\title{
Abnormal brain scan with subacute extradural haematomas
}

\author{
J. BARRIE MORLEY AND KEITH H. LANGFORD \\ From the Departments of Neurology and Neurosurgery, and the Radio-Isotopes Department, \\ Prince Henry's Hospital, Melbourne, Australia
}

SUMMARY Four patients are described with proven subacute extradural haematomas, each with an abnormal cerebral scan of diagnostic assistance. A possible mechanism of production of the subacute extradural haematoma is discussed, and appears to be similar to the mechanism involved in the subacute subdural haematoma. The means by which the abnormal scan results in such cases is also examined, from which it appears that non-specific meningeal membrane inflammatory reaction surrounding the haematoma is significant.

The recent developments in radio-isotopic cerebral scanning have been devoted substantially to the detection of tumours, infarctions, and haemorrhages. However, one of its intriguing features is the scan's ability to demonstrate a wide variety of different pathologies, and there is much interest in the pathophysiological mechanisms responsible for an abnormal scan appearance. Several studies (for example, Matthews and Molinaro, 1963; Mundinger, 1965; Tator and Olszewski, 1966; Wagner and Holmes, 1968) have delineated the main factors, which can be summarized as follows: (1) circulating isotope being present in the increased vascular pattern associated with the lesion; (2) breakdown of bloodbrain barrier, with increased capillary permeability admitting isotope into the interstitial space associated with the lesion; (3) occasionally specific uptake by neoplastic cells.

Different mechanisms would seem to predominate according to either the type of lesion or the type and preparation of radio-isotope employed.

Recently there has been opportunity in these departments to scan four patients with proven subacute extradural haematomas. Although one patient's scan interpretation was complicated by a previous exploratory burr hole, and another's by an associated subgaleal haematoma, in each the scan was abnormal. These patients are reported briefly for two reasons: not only are the scan results of interest in this condition, but they highlight some questions of the pathophysiology involved in the abnormal brain scan.
Standard rectilinear scanning technique was employed, after injecting 15-18 mCi Tc-pertechnetate, by scanning with a Picker Magna Scanner, using a conventional collimator (Cat. 2111) with a 5 in. crystal. Before the procedure potassium perchlorate 200 to $400 \mathrm{mg}$, according to the age of the patient, was administered orally.

CASE 1

(PHH 19974) G.L.S., an 11 year old boy, was transferred from another hospital six days after he was struck by a cricket ball on the left side of his head. The blow stunned him but he remained conscious. Within the next half-hour he became drowsy, in which fluctuating state he continued. He also complained of recurring severe frontal headache accompanied by vomiting.

After five days he was admitted to the local country hospital where his drowsiness increased. Mild swelling of the left optic disc and an extensor right plantar response were noted. Accordingly, his transfer was arranged for neurosurgical management.

On admission he was orientated but drowsy; with mild bilateral papilloedema and a mild right spastic hemiparesis. A left carotid angiogram apparently was normal. This then raised the possibility that the antecedent head injury might have been unrelated, and that he had some form of infiltrating neoplasm. Accordingly, an upper left frontal burr hole was made (Figs. 1 and 2), with the intention of proceeding to ventriculography. However, the brain was found to be slack and there was no subdural haematoma; a brain biopsy was taken which was found to be normal.

For several days his condition remained unchanged. On the sixth day of the admission brain scan was per- 
FIG. 1. Post-operative anteroposterior skull radiograph of case 1 , showing original burr hole $(B)$, defect of subsequent craniotomy, and its associated irrigation holes.

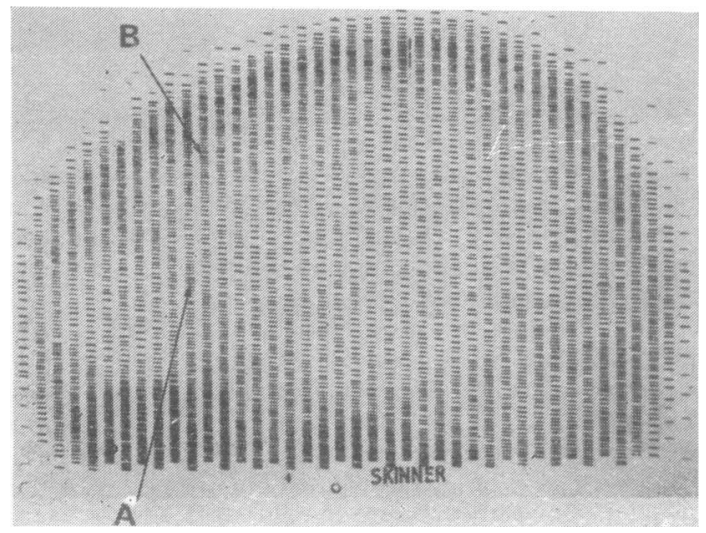

FIG. 3. Left lateral view of scan of case 1, showing arcuate line of increased uptake in the frontal region $(A)$, and increased uptake due to prior burr hole $(B)$.

formed (Figs. 3 and 4). This demonstrated an abnormal 'arcuate rim' appearance in the lateral view, extending upwards and forwards from the inferior frontal region; in the anterior view this was seen as a diffuse change

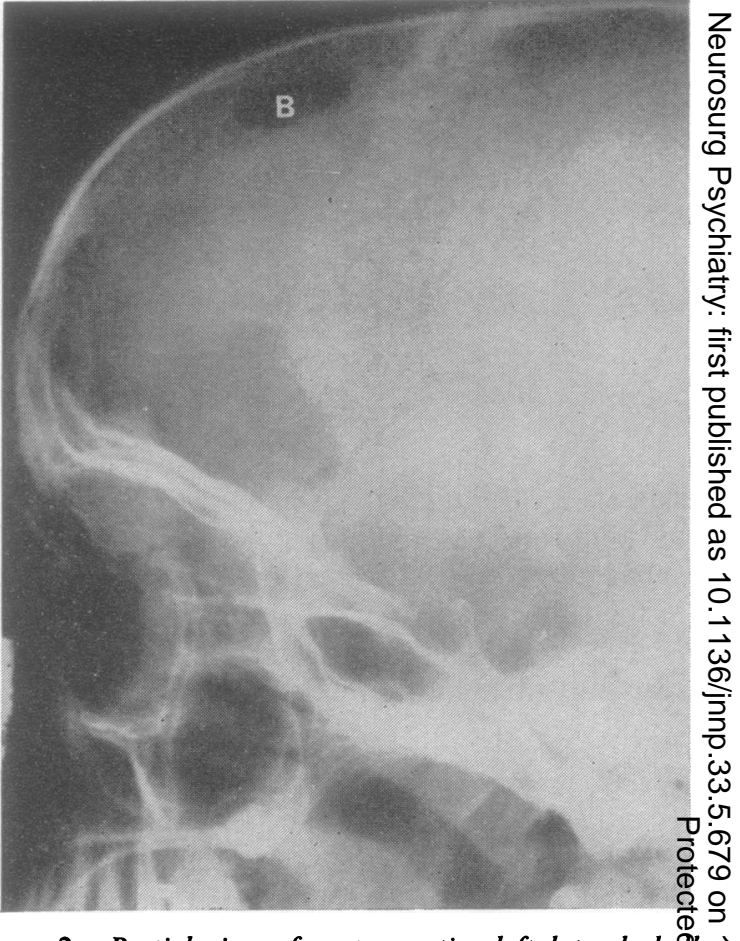

FIG. 2. Partial view of post-operative left lateral skuft radiograph of case 1, again showing original burr hote $(B)$ and subsequent craniotomy defect.

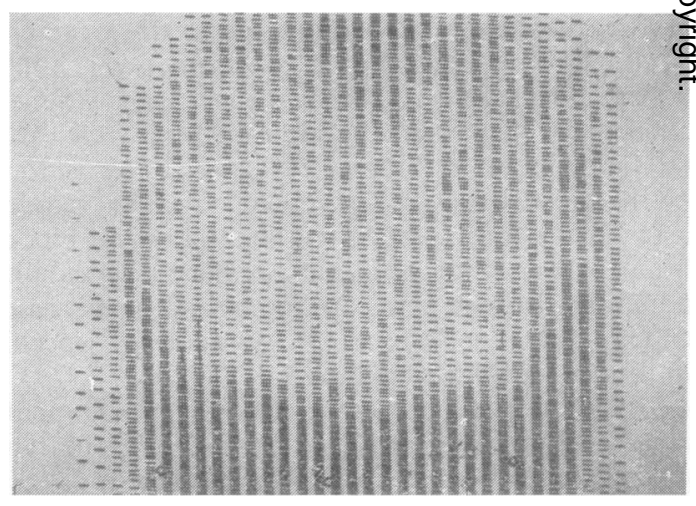

FIG. 4. Anterior view of scan of case 1, showing a diffuse area of increased uptake, in the lateral region of the left frontal area.

throughout most of the lateral left frontal area.

Frontal craniotomy was then performed at which was found a small fracture of the frontal sinus, and behind it was an extensive extradural haematoma passing over $D$ most of the anterior pole of the left frontal lobe. As part of the operative procedure two small frontal holes also $\mathrm{N}$ 
were placed for irrigation purposes. After the extradural haematoma was evacuated the patient made an uneventful recovery.

\section{CASE 2}

(PHH 45213) J.M., a 13 year old girl, was admitted three days after sustaining head and other injuries when she fell through a roof skylight. She retained consciousness but over the next few hours became drowsy, began to vomit, and complained of severe headache. Her pulse rate dropped to 50 per minute and within the next 24 hours her pupils became unequal.

On admission she was drowsy. A right periorbital haematoma was evident and the only neurological finding was her pupillary inequality, the left being larger than the right; both reacted sluggishly. Radiographs of the skull disclosed a fracture of the mandible, just to the right of the midline, but there was no skull fracture. Right carotid angiograms (Figs. 5 and 6), revealed displacement of the anterior cerebral artery to the left of the midline, with separation of the pericallosal and calloso-marginal branches, interpreted as being suspicious of a localized frontal or anterior temporal subdural or extradural haematoma.

Over the next two days her condition improved in that she was less drowsy, and the pupils reverted to normal. However, a brain scan was performed on the third day of her admission (Figs. 7 and 8) to demonstrate increased uptake in the right frontotemporal region. Consequently, right temporal and frontal burr holes were performed; when her head was shaved, a small subgaleal haematoma in the right temporal region was evident. On entering the cranium a moderate sized extradural haematoma was found extending over the anterior and lateral aspects of the temporal lobe, passing forward and anteriorly into the anterior cranial fossa; a total of $30 \mathrm{ml}$. of dark blood was aspirated. The presence of a fracture of the right sphenoid lesser wing was also noted. Her post-operative course was complicated by the persistence of raised intracranial pressure, marked by mild papilloedema and bilateral sixth nerve pareses. She responded to parenteral dexamethasone and when eventually discharged, six weeks after admission, she had only minor residual abducens weaknesses.

\section{CASE 3}

(PHH 38750) J.B., a 24 year old male, was transferred from another hospital five days after he was struck on the head by a bottle during a fight. Gradually he became drowsy and two days later was admitted to the local hospital. He was disorientated and over the next two days developed dysphasia and perseveration, with a mild right hemiparesis. Accordingly, he was transferred for neurosurgical management.

On admission, there were no further findings than the hemiparesis and the speech disturbance. Skull radiographs were normal but bilateral carotid and vertebral angiographic studies (by right femoral catheter) demonstrated marked displacement of both anterior cerebral arteries to the right of the midline (Fig. 9). On the brain scan there was abnormal uptake in the left frontotemporal region (Figs. 10 and 11).

On the sixth day of admission, left frontal burr holes were performed at which was found an extradural haematoma from which a total of $50 \mathrm{ml}$. was aspirated. Thereafter he made an uneventful recovery.

\section{CASE 4}

(QVH 087773) A.S., a 27 year old male, was referred from another hospital for brain scan with the following history. He was born in Ceylon and had migrated to Australia two years before this illness. When aged 14 he had sustained head injuries in a car accident after which he suffered recurrent major convulsions which had remained under fair control with anti-convulsant therapy. Meanwhile, he developed increasing problems of chronic alcoholism. Because of his alcohol indulgence and his periodic seizures, at various times he sustained head injuries of varying severity.

He presented to hospital in coma but without external evidence of recent trauma, and the neurological examination disclosed only a left extensor plantar response; there was no papilloedema. During his admission examination he had a brief right focal fit. Over the next few hours he regained full consciousness, but his mental state remained vague for a few more days. In addition, bilateral periorbital haematomas appeared.

Skull radiographs demonstrated a stellate fracture of the vertex with slight depression of the parietal bone at the apex of the fracture. Lumbar puncture disclosed clear colourless fluid with only a few crenated red blood cells and a normal protein level $(30 \mathrm{mg} / 100 \mathrm{ml}$.)

Ten days later it was noted that he now had developed early papilloedema, despite continued slow improvement in his general condition. On the fifteenth day of admission a brain scan demonstrated a striking abnormality (Figs. 12, 14,15); the pcsterior view appearance (Fig. 14) was considered similar to that of a 'butterfly glioma.' Subsequently, right carotid angiography (Figs. 13 and 16) revealed this to be due to a considerable downward displacement of the superior sagittal sinus, marked by an extensive avascular area over the cerebral surface in the midline.

By this time papilloedema had almost completely receded again and his clinical state remained very satisfactory. For some weeks he was kept under observation, but eventually a burr hole exploration was made, using biparietal holes $2 \mathrm{~cm}$. from the midline. When the scalp had been reflected, it was seen that the thickened periosteum showed a localized recent haemorrhage near to which was the coronal fracture which itself did not appear recent. No recent haemorrhage was found beneath the burr hole, but a brown felted membrane $w$ as seen. This membrane punctured readily to allow the escape of watery yellow fluid with a small amount of darker fluid posteriorly in the same cavity. Membrane formation was extensive but no calcification was seen. Pressure was only slightly raised and about $100 \mathrm{ml}$. of fluid were evacuated. The cavity was drained and the patient's recovery was uneventful. 


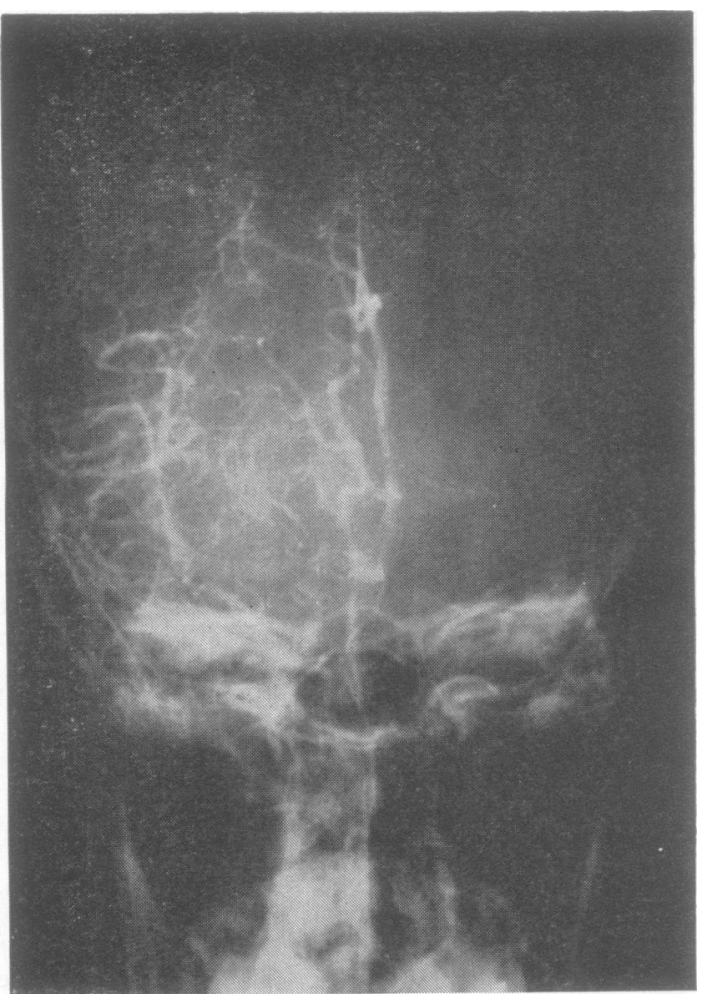

FIG. 5. Anteroposterior projection of right carotid angiogram of case 2, demonstrating displacement of the anterior cerebral artery to the left of the midline.

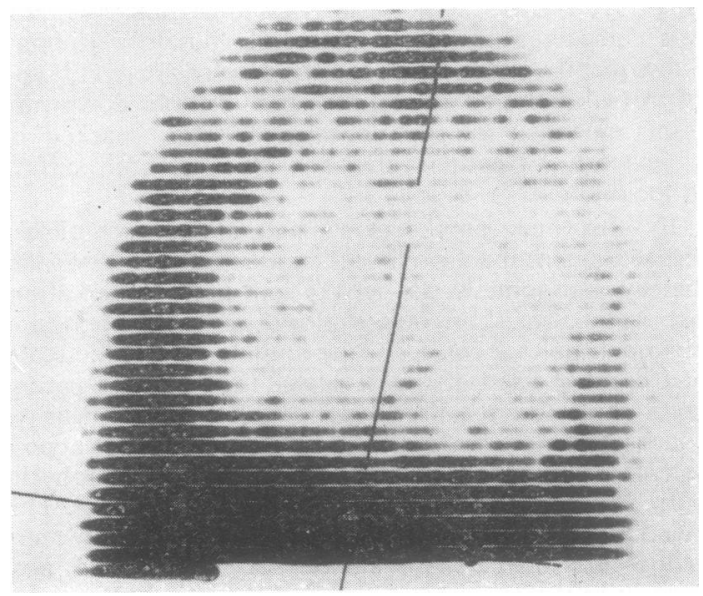

FIG. 8. Anterior view of cerebral scan of case 2, again demonstrating the diffusely increased uptake in the right frontal and temporal regions.

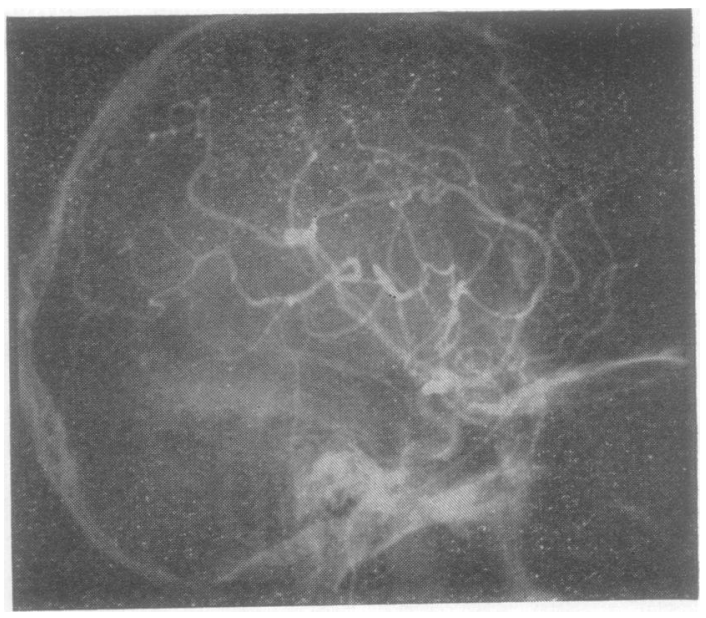

FIG. 6. Right lateral view of right carotid angiogram of case 2 .

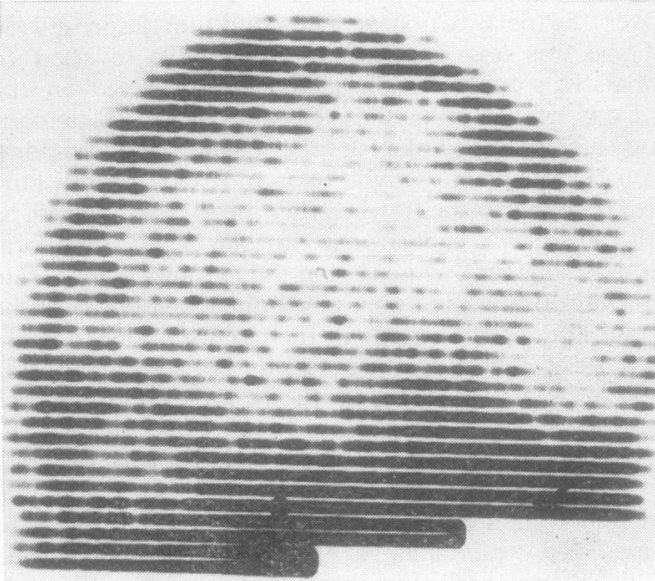

FIG. 7. Right lateral view of cerebral scan of case 2, demonstrating patchy and diffusely increased uptake in the right fronto-temporal region.

\section{DISCUSSION}

From the diagnostic viewpoint, although there is no specific scan appearance, it is noteworthy that, in each of these four cases, the brain scan was abnormal. It is believed that this is the first report of the abnormal brain scan with subacute extradural haematoma.

With case 1 , the preliminary superior frontal $\frac{\text { D }}{2}$ burr hole was situated at some distance from the $N$ site of the brain scan abnormality and indeed in the lateral view of this scan (Fig. 3) the increased 0

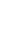

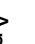

,




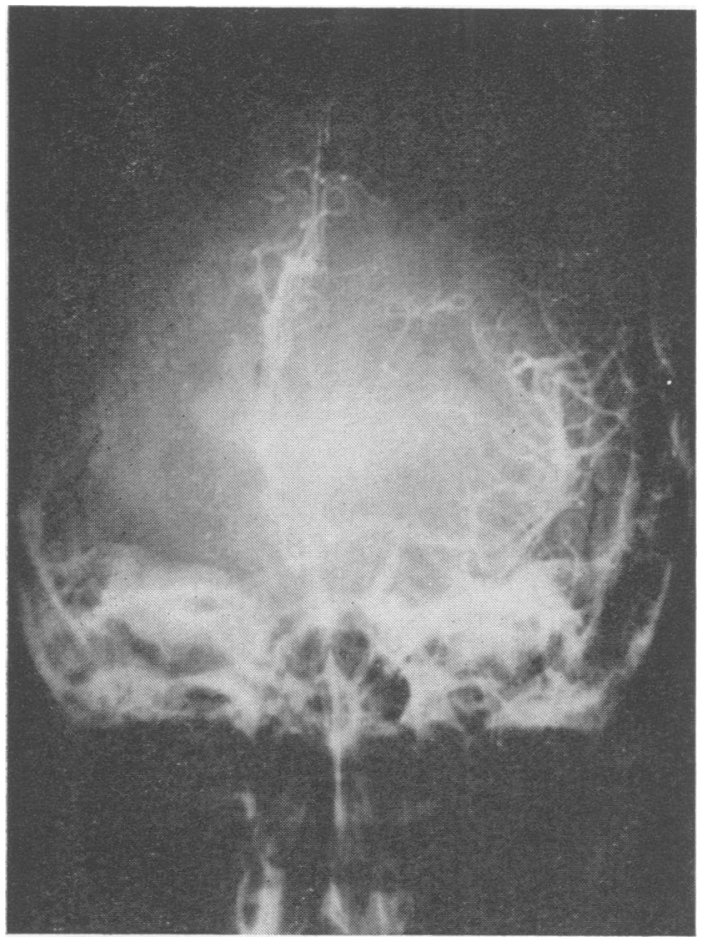

FIG. 9. Anteroposterior projection of left carotid angiogram of case 3, demonstrating marked curving of both anterior cerebral arteries to the right of the midline.

uptake due to this burr hole can be seen as a separate feature from the appearance which is presumed to be due to the subacute extradural haematoma. Comparison of the scan with the post-operative radiographs of the skull (Figs. 1 and 2) assists further to document this. Similarly, this separation renders unlikely another possible objection, that the

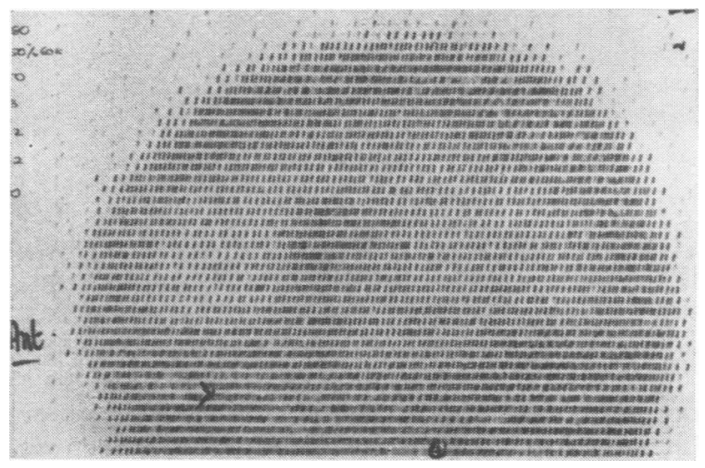

FIG. 10. Left lateral scan of case 3, showing abnormal uptake in the left posterior frontal region.

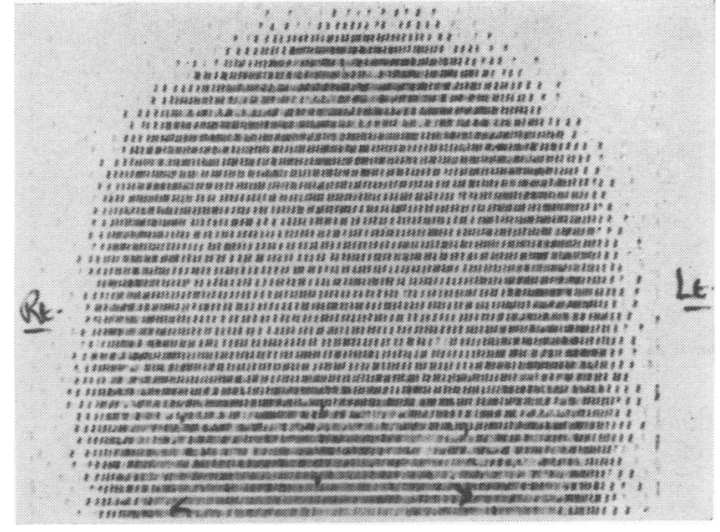

FIG. 11. Anterior view of cerebral scan of case 3, demonstrating the abnormal uptake fairly superficially placed in the left frontal region.

extradural haematoma might have originated in the making of the preliminary burr hole.

In case 2 , it is probable that the subgaleal haematoma contributed to the abnormal picture, for it is well established that these pericranial pathologies can produce an abnormal scan. However, in view of the minor extent of this superficial haematoma, it seems difficult to account for this alone in the extensive scan abnormality, especially that of the anterior view (Fig. 8). Moreover, the distribution of the increased uptake in this patient's scan conformed well with the operative findings of the extradural haematoma.

In case 4 , the designation of the extradural haematoma as 'subacute' at the time of its scanning is semi-arbitrary. It is a reasonable surmise that the head injury which initiated the patient's extradural haematoma occurred shortly before his admission: he was admitted to hospital in coma, to recover shortly after; several days later bilateral periorbital haematomas developed, and over the first 10 days of his admission there gradually developed bilateral papilloedema. Presumably, when the scan was performed on the fifteenth day of admission the extradural haematoma was of 'subacute' nature, if the term 'subacute' is used with that time limit as employed in distinguishing the same subdural haematoma from its 'chronic' counterpart. Of course, by the time the patient came to neurosurgical exploration, by this definition it was now of 'chronic' nature, which would generally be supported by the operative findings of a dark felted membrane and the enclosed watery yellow fluid.

The striking appearance of the posterior brain scan view of case 4 deserves brief comment. In retrospect, the original suggestion of 'butterfly 


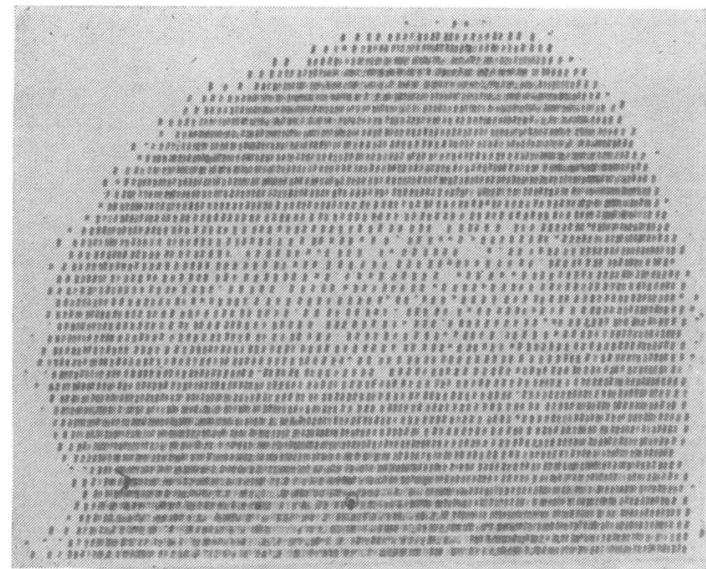

FIG. 12. Left lateral scan of case 4, showing diffuse appearance of abnormal uptake across most of the superior regions of the hemisphere.

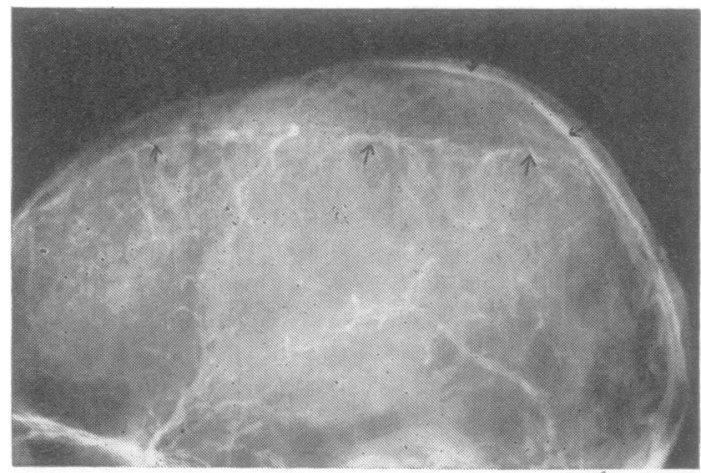

FIG. 13. Part of lateral view of carotid angiogram (venous phase) of case 4, again showing the 'bare area' of the extradural haematoma.

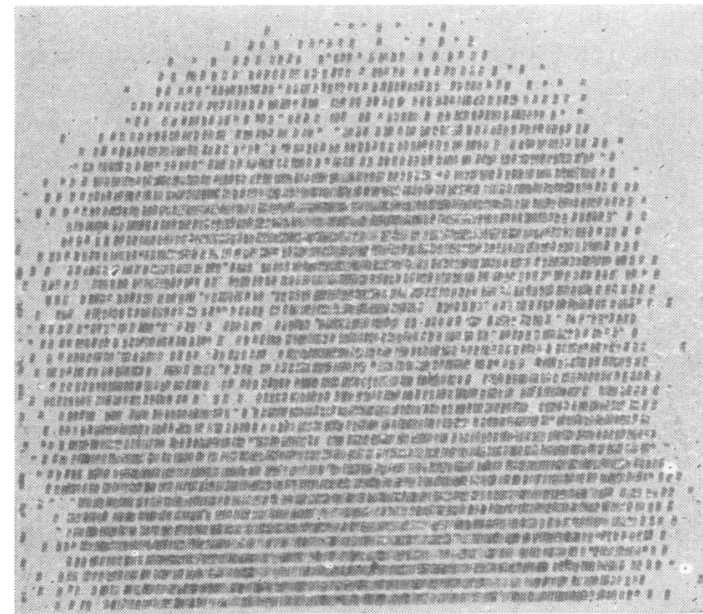

FIG. 14. Posterior scan of case 4, revealing the similarity of appearance in this view to that of 'butterfly glioma'.

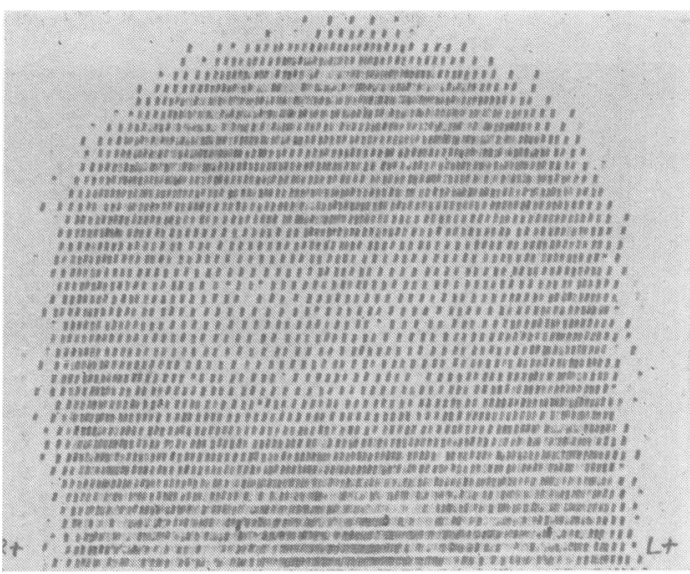

FIG. 15. Anterior scan of case 4, again demonstrating the diffuse and bilateral nature of the uptake.

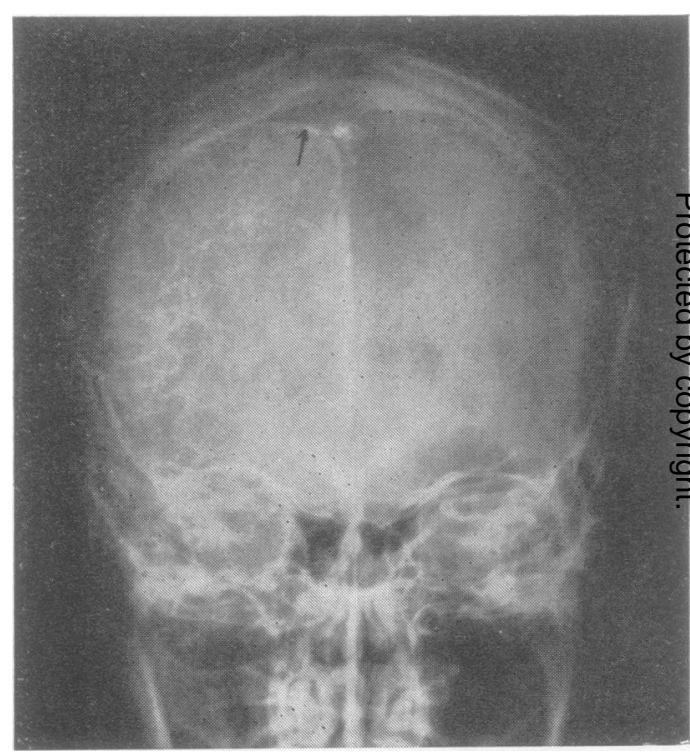

FIG. 16. Anteroposterior right carotid angiogram (venous phase) of case 4, demonstrating downward displacement of superior sagittal sinus.

glioma' can be seen to be derived from the radiating impression given by the downward displacement of the superior sagittal sinus and its immediately $\frac{3}{3}$ adjacent cortical structures-in its way a gross $\bigcirc$ 'arcuate rim' appearance - thus simulating an ab- $\supset$ normality within the corpus callosum extending $\frac{D}{0}$ laterally into both hemispheres. The pertinent clue is that in this view, the sinus is not evident at the vertex. Moreover, the lateral view is not truly consistent with 'butterfly glioma' which would beN 
expected to show as a fairly localized and intense abnormality from the lateral aspect.

It may be added that, in diagnostic value, not only did the scanning procedure assist to substantiate the diagnosis in all four of these patients, but it was of considerable aid in case 1 , in whom the presence of the haematoma could not be established clearly by the carotid angiogram two days before the scan. Also, in case 2 there is no doubt that the scan result influenced the decision to proceed to surgical exploration at that time.

It is noteworthy that all scans were performed a week or more after the initiating head injury.

It may also be of interest for some remarks to be made regarding the manner in which an abnormal scan is produced in these patients. This introduces questions of the pathogenetic mechanisms involved in the causation of the subacute extradural haematoma, and of the pathophysiological processes by which such a wide variety of cerebral lesions produce an abnormal brain scan.

With regard to the first of these questions, clinically the illnesses of all four of these patients were indistinguishable from those of subacute subdural haematoma, and, of course, these two conditions share many pathological similarities. However, the subacute and chronic extradural haematomas are rare conditions, and one of the authors (K.H.L.) has not seen a chronic extradural haematoma previously in 12 years of experience with trauma. This prompts the question as to why this is so, when subacute and chronic subdural haematomas are so numerous. Limitation of expansion by the dural adhesions to bone at the margin of the cavity might provide an answer, the brain being capable of much greater deformation than the dura mater. Alternatively, the membrane formation may occur much more readily between the meninges than in the extradural space. Apart from these distinctions, it does not seem unreasonable to assume that the same mechanisms are involved in the development of subacute-chronic extradural haematomas as in their subdural equivalents. Expansion of the subacute and chronic subdural haematoma is said to be caused by an increasing osmolarity of the fluid, due to the build-up of albumin from the abnormally permeable blood vessels in the highly vascular membrane surrounding the collection (Gitlin, 1955; Rabe, Flynn, and Dodge, 1962). The rapid transfer from the blood stream into the fluid collection of radioactive iodine tagged albumin (Rabe, Young, and Dodge, 1964) lends support to this theory. In the subacute and chronic extradural haematoma, a similar state of abnormal permeability may exist in the membrane or in the vessels of the dura mater beneath the clot. It is readily apparent that these considerations deserve attention when examining the possible means by which these extracerebral collections produce an abnormal scan. Already, it is well established that at times a subdural haematoma can be detected by the brain scan (Mealey, 1963; Ciric, Quinn, and Bucy, 1967), and other previous cases have been reviewed briefly by Cowan, Maynard, and Lassiter (1970).

Any hypothesis of the causation of an abnormal brain scan must be consistent in explanation of all of those scanning phenomena which are peculiar to cerebral scanning. It must explain why lesions are demonstrated by increased rather than decreased uptake, why a number of unrelated isotopes can demonstrate the presence of pathology, why a wide variety of pathologies are demonstrable, and why intracerebral, extracerebral, and pericranial pathologies all may be detected. From the above, for subdural and extradural haematomas, it seems probable that there are involved the properties of the vascularity of the membrane surrounding the collection; any accumulation of isotopes within the collection itself is essentially a direct result of this membrane vascularity. Cowan et al. (1970) suggest that the accuracy of diagnosis of subdural haematoma is greater in cases which are older than 10 days, which seems consistent with the hypothesis of an association with the development of the membrane. These authors regard the membrane as being responsible but they cannot decide if the uptake is mainly in the membrane or is largely in the fluid. Others have noted $\mathrm{Hg}$ chlormerodrin in subdural fluid (Kramer and Rovit, 1964; Williams and GarciaBengochea, 1965). On the other hand, Gilson and Gargano (1965) have produced experimental evidence that in dogs there is a significantly higher uptake of radioactive mercury in the membrane than in the fluid. In two of the patients of this report, an inconclusive attempt was made to examine these matters further: at operation, blood from the extracranial vessels was compared with fluid from the clot cavity. In case 1 , the count was higher in the clot fluid, but there was little difference in the respective counts for case 2 . Nevertheless, on the appearance of the posterior view of case 4 (Fig. 14), there is a striking visual impression that, at least in this case, it was the membrane rather than the fluid which contributed to the abnormal picture.

However, it may be redundant to attempt to decide whether it is either membrane property or accumulation of isotopes within the fluid collection which produced the abnormal scan appearance. Both of these possibilities potentially could be equally applicable, if it can be accepted that accumulation of the isotope within the fluid collection is a direct result of membrane vascularity and permeability 
(Rabe et al., 1964). Indeed, it may be expected that the more longstanding is the extradural or subdural haematoma, the more likely it will be that membrane factors will become subjugate to the factor of isotope accumulation within the fluid collection in the production of the abnormal scan. In these circumstances, a longer-lived isotope would be anticipated to present better this cumulative collection effect than would the shorter-lived isotopes currently in common use for brain scanning.

Furthermore, such a concept of basic dependence upon membrane activity is consistent with the reasonable speculation of the causation of any abnormal brain scan, and one which explains all those features to which attention was drawn above. This speculation can be derived from drawing together two of the postulated fundamental mechanisms, as summarized in the introduction of this paper. Increased tissue vascularity and breakdown of capillary-interstitial space barrier (blood-brain barrier), either of the lesion itself or of the surrounding tissue, are two of the integral processes involved in any tissue's inflammatory response. Such a nonspecific concept could apply whether the pathological process were present in cerebral tissue, in meningeal tissue, or in the pericranium. Indeed, these considerations apply theoretically to other organs and tissues; but they are of practical import only so far as the head is concerned, for this is the one body region where there is a significant volume of adjoining (or 'background') tissue which is relatively impermeable to isotopes, and of relatively low vascularity.

This explanation, of dependence on basic inflammatory reactions, seems readily applicable to the abnormal brain scan of the subacute subdural haematoma, and by assumption, that of the subacute extradural haematoma as well. Thus, it can be envisaged that, at least after several days, there would be sufficient meningeal activity to provide 'increased uptake' of the isotope in the vicinity of the clot, and within the collection itself.

Finally, in terms of clinical practice, these cases serve to illustrate that the rectilinear cerebral scan can be employed, usually on a quiet patient, to aid the detection of a subacute and chronic extradural haematoma, and the occurrence of positive scans in these cases makes this investigation a reasonable alternative to angiography, especially in children. A gamma camera is a more attractive investigation since the time involved, being less, compares favourably with angiography. A restless or anxious patient may be controlled by neuroleptic drugs as is common practice for angiography. However, the apparently greater incidence of false negative scans in the acute cases of extracerebral haemorrhage stilis makes angiography the investigation of choice in these instances.

Mrs. D. Morley, and the staff of the Radio-Isotopes? Department, rendered invaluable technical assistance Mr. J. Scrimegour and Mr. H. Berkshire, hospita $\bar{R}$ photographers, at Prince Henry's Hospital and the Queen Victoria Memorial Hospital respectively, pres⿱乛龰 pared the illustrations. Dr. D. A. Coventry kindly gaveo permission for publication of case 4 .

\section{REFERENCES}

Ciric, I. S., Quinn, J. L., and Bucy, P. C. (1967) Mercury 197 and Technetium $99 \mathrm{~m}$ brain scans in the diagnosiss of non-neoplastic intracranial lesions. J. Neurosurg. $\vec{b}$ 27, 119-125.

Cowan, R. J., Maynard, C. D., and Lassiter, K. R. (1970) Technetium-99m pertechnetate brain scans in the detec $-\bar{W}$ tion of subdural hematomas: a study of the age of the lesion as related to the development of a positive scan J. Neurosurg., 32, 30-34.

Gilson, A. J., and Gargano, F. P. (1965). Correlation of brain scans and angiography in intracranial trauma. Amere J. Roentgenol., 94, 819-827.

Gitlin, D. (1955). Pathogenesis of subdural collectionsक्षे fluid. Pediatrics, 16, 345-352.

Kramer, S., and Rovit, R. L. (1964). The value of $\mathrm{Hg}$ 舫通 brain scans in patients with intracranial hematomas.Radiology, 83, 902-909.

Matthews, C. M. E., and Molinaro, G. (1963). A study the relative value of radioactive substances used for broing tumour localization and of the mechanism of tumar@ brain concentration. Uptake in transplantable fibjosarcoma, brain and other organs in the rat. Brit. J. expse Path., 44, 260-277.

Mealey, J. (1963). Radio-isotopic localization in subdural hematomas. J. Neurosurg., 20, 770-776.

Mundinger, F. (1965). The biological bases of the utilization of radio-isotopes for gamma-encephalography. In Radio Isotopes et Affectiones du Systeme Nerveux Central, pp. 73. 87. Edited by T. Paniol. Masson: Paris.

Rabe, E. F., Flynn, R. E., and Dodge, P. R. (1962). A stud of subdural effusions in an infant. With particular reference to the mechanisms of their persistence. Neurology (Min? neap.), 12, 79-92.

Rabe, E. F., Young, G. F., and Dodge, P. R. (1964). The distribution and fate of subdurally instilled human serum? albumin in infants with subdural collections of fluid. Neurology (Minneap.), 14, 1020-1028.

Tator, C. H., and Olszewski, J. (1966). Factors responsible for the distribution of radioactivity in a mouse glioma and brain after injection of radioiodinated human serunp. albumin (R.I.H.S.A.). Cancer Res., 26, 1569-1581.

Wagner, H. N., and Holmes, R. A. (1968). The nervous system. In Principles of Nuclear Medicine, p. 655. Edited by $H$. N. Wagner. W. B. Saunders: Philadelphia.

Williams, C. M., and Garcia-Bengochea, F. (1965). Concen tration of radioactive chlormerodrin in the fluid of chronis subdural haematoma. Radiology, 84, 745-747. 